\title{
Takotsubo Cardiomyopathy in Patients Suffering From Acute Non-traumatic Subarachnoid Hemorrhage - A Single Center Follow-up Study
}

Csilla Molnár

Debreceni Egyetem

Judit Gál

Debreceni Egyetem

Dorottya Szántó

Debreceni Egyetem

László Fülöp

Debreceni Egyetem

Andrea Szegedi

Debreceni Egyetem

Péter Siró

Debreceni Egyetem

Endre V Nagy

Debreceni Egyetem

Szabolcs Lengyel

Magyar Tudományos Akadémia Ökológiai Kutatóközpont: Okologiai Kutatokozpont

János Kappelmayer

Debreceni Egyetem

Béla Fülesdi ( $\square$ fulesdi@med.unideb.hu )

University of Debrecen https://orcid.org/0000-0003-2541-9740

Research

Keywords: subarachnoid hemorrhage, takotsubo cardiomyopathy, outcome

Posted Date: September 20th, 2021

DOI: https://doi.org/10.21203/rs.3.rs-889430/v1

License: (1) (1) This work is licensed under a Creative Commons Attribution 4.0 International License.

Read Full License 


\section{Abstract}

Background: Takotsubo cardiomyopathy (TTC) is an important complication of subarachnoid hemorrhage (SAH), that may delay surgical or endovascular treatment and may influence patient outcome. This prospective follow-up study intended to collect data on the prevalence, severity, influencing factors and long-term outcome of TTC in patients suffering from non-traumatic SAH.

Methods: Consecutive patients admitted with the diagnosis of non-traumatic SAH were included. Intitial assessment consisted of cranial CT, Hunt-Hess, Fisher and WFNS scoring, 12-lead ECG, transthoracic echocardiography (TTE), transcranial duplex sonography and collecting laboratory parameters (CK, CKMB, cardiac troponin T, NT-proBNP and urine metanephrine and normetanephrine). Diagnosis of TTC was based on modified Mayo criteria. TTC patients were dichotomized to mild and severe forms. Follow-up of TTE, Glasgow Outcome Scale assessment, Barthel's and Karnofsky scoring occurred on days 30 and 180.

Results: One hundred thirty six patients were included. The incidence of TTC in the entire cohort was 28.7 $\%$; of them, $20.6 \%$ and $8.1 \%$ were mild and severe, respectively. TTC was more frequent in females (30/39; $77 \%)$ than in males $(9 / 39 ; 23 \%)$ and was more severe. The occurrence of TTC was related to mFisher scores and WFNS scores. Although the severity of TTC was related to mFisher score, Hunt-Hess score, WFNS score and GCS, multivariate analysis showed the strongest relationship with mFisher scores. Ejection fraction differences between groups were present on day 30, but disappeared by day 180 , whereas wall motion score index was still higher in the severe TTC group at day 180 . By the end of the follow-up period (180 days), 70 (74.5\%) patients survived in the non-TTC, 22 (81.5\%) in the mild TTC and $3(27 \%)$ in the severe TTC group $(n=11)(p=0.002)$. At day 180 , GOS, Barthel, and Karnofsky outcome scores were higher in patients in the control (non-TTC) and the mild TTC groups than in the severe TTC group.

Conclusions: Takotsubo cardiomyopathy is a frequent finding in patients with $\mathrm{SAH}$, and severe TTC may be present in $8 \%$ of SAH cases. The severity of TTC may be an independent predictor of mortality and outcome at 6 months after disease onset. Therefore, a regular follow-up of ECG and TTE abnormalities is warranted in patients with subrachnoid hemorrhage for early detection of TTC.

Trial registration: The study was registered at the Clinical Trials Register under the registration number of NCT02659878 (date of registration: January 21, 2016)

\section{Introduction}

First described in a case series of Japanese patients in 1991, Takotsubo cardiomyopathy (TTC) represents a reversible left ventricular dysfunction, that mimicks acute coronary syndrome, without the evidence of obstructive coronary artery disease (1). In the past 3 decades several synonimes were used for TTC, including „apical balloning”, „stress-induced cardiomyopathy”, „broken heart syndrome” and „transient ventricular ballooning syndrome” (2). Its estimated incidence is 100 new cases per 1 million inhabitans per year (3) and the prevalence of the syndrome ranges from 1.7 to $2.2 \%$ among patients 
admitted with suspected coronary artery syndrome (4). The most important causative factors are intense emotional events and any form of physical stress, including severe medical illness. Neurological emergencies represent a large group of the underlying diseases; among them most frequent are subarachnoid hemorrhage, ischemic stroke and epileptic status (5-7). Subarachnoid hemorrhage (SAH) is the most common neurological cause, making up one-third of the cases. (8). The prevalence of TTC in SAH ranges from $1.2 \%$ up to $26 \%$ in different studies (9-12). The relatively wide range of the prevalence might be in part explained by the changing definition of TTC over the past decades. Recent evidence suggest that prospective studies using predetermined time intervals for echocardiography and taking apical and mild cases also into account may be necessary to evalute the real incidence (13-15). The importance of TTC in SAH is underlined by the fact that it may lead to neurogenic pulmonary edema, necessitate hemodynamic and ventilatory support and thus has the potential to hamper the treatment especially in severe subarachnoid hemorrhage cases with cerebral vasospasm or raised intracranial pressure. Additionally, TTC may delay surgical or endovascular treatment. Up till now, the majority of the knowledge about the natural history of TTC among patients with SAH has been gathered from retrospective analysis of databases. Prospective long-term follow-up studies on the prevalence and clinical outcome of TTC are scarce.

In the present study we collected data prospectively on the prevalence, severity, influencing factors and long-term outcome of TTC in patients suffering from non-traumatic subarachnoid hemorrhage.

\section{Subjects And Methods}

This prospective follow up study was performed at the 8-bed neurosurgical ICU of the Department of Anesthesiology and Intensive Care, University of Debrecen. The ICU serves as a regional center for managing acute subarachnoid hemorrhages being responsible for approximately 2 million inhabitants. During the period between March 2017 and December 2018, all patients who were admitted for SAH were checked for eligibility. The study was registered at the Clinical Trials Register under the registration number of NCT02659878 (date of registration: January 21, 2016). The protocol was approved by the Institutional Ethics Committee of the University of Debrecen (registration number: DE RKEB/IKEB:4317 2015). All included patients or their closest relatives gave written informed consent before participation.

Inclusion criteria were acute subarachnoid hemorrhage (SAH) in adult (age $>18$ years) patients, provided that the patient arrived at the hospital not later than 48 hours after symptom onset. Patients with head trauma, angioma or A-V malformations were excluded. Patients with known myocardial diseases, including myocardial infarction, heart failure, known structural heart diseases (severe, clinically significant valve insufficiency, and / or significant stenosis) preexisting myocarditis, known coronary artery stenosis necessitating ballon angioplasty, hypertrophic cardiomyopathy and phaechromocytoma were excluded.

Decision on the treatment modality in the acute phase (coling or surgical clipping) was based on consultation with the neurosurgeon and neurointerventionist. Non-surgical and non-interventional therapy 
at the ICU was performed according to the local protocol that includes maintenance of normovolemia, normocapnia, normoxemia, normomagnesemia and normoglycemia. The use of nimodipine $(6 \times 60 \mathrm{mg}$ per os) and analgosedation was based on international guidelines (16). During the course of the disease, surgical interventions or placing intraventricular or lumbar drains were performed as required.

The diagnosis of TTC was based on the modified Mayo criteria (17), taking the following aspects into account:

- Transient LV/RV wall motion abnormalities, usually extending beyond a single epicardial coronary artery distribution

- Evidence of myocardial involvement by biomarker elevation: cardiac troponin I, creatine kinase, brain natriuretic peptide; $\mathrm{N}$-terminal prohormone of brain natriuretic peptide

- ECG abnormalities: ST elevation/depression, negative T-waves, new bundle brunch blocks

- Potential coronary artery culprit ruled out

Diagnosis of TTC was first verified or excluded in each SAH patient by performing the investigations listed above within 24 hours after admission.

On admission and on the subsequent 6 days (days 1 trough 7) the following investigations were performed on a daily basis: 12-lead ECG; cardiac biomarkers (cardiac troponin T, creatine kinase, $\mathrm{N}$ terminal prohormone of brain natriuretic peptide), transcranial color-coded duplex sonography with blood flow velocity measurements, Glasgow Coma Score (GCS). World Federation of Neurological Society (WFNS) scores, Fisher and Hunt-Hess grading were performed at admission. Collection of urine for determination of urine metanephrine and normetanephrine levels, and transthoracic echocardiography was performed on day 1 and day 7. If new ECG abnormalities occured between days 1 and 7, additional echcardiography was performed immediately.

During the 30 day follow up visit the following diagnostic measures were taken: transthoracic echocardiography; 12-lead ECG; New York Heart Association score (NYHA score) assessment; Glasgow Outcome Scale assessment; Barthel's and Karnofsky indices assessments. Additionally, patients were asked to perform a 24-hours timed collection of urine for determination of urine metanephrine and normetanephrine levels one day prior to coming for checkup. If cardiac wall motion abnormality was present on echocardiography, cardiac stress test, CT coronary angiography or conventional coronary angiography were performed as required.

During 180 day follow up visit the same tests and examinations were performed as on day 30 .

Transthoracic echocardiography was performed by two experienced cardiologists (LF, ASz) by Mindray TE7 device (P4-2s 3.5 MHz harmonic imaging transducer) at the initial hospitalization and during followup. Investigators were unaware of patient's other parameters (laboratory and ECG results referring to TTC), they only knew that the patient suffered from SAH. During echocardiographic evaluation in 2Dmode the parasternal short axis and apical two- and three-chamber views have been visualized to 
evaluate left ventricular systolic and end diastolic diameters and wall motion abnormalities. Ejection fraction was calculated by Simpson's equation.

According to the international guidelines the diagnosis of TTC was based on transthoracic echocardiography. This is merely based on two factors: hypo- or akinesis of at least one or more segments of the heart and decreased ejection fraction. The wall motion abnormalities were defined by using the 16-segment model. Segmental wall motion abnormalities have been scored according to the usual method: 1- normokinesis, 2- hypokinesis, 3-akinesis, 4 -dyskinesis, 5 -aneurysm. In order to describe the number and the severity of the affected myocardial regions, the wall motion score index (WMSI) was calculated by dividing the total of the wall motion scores of each segment by 16. (18). We also took the ejection fraction into account for the differentiation between mild ( $E F \geq 40 \%)$ and severe TTC (EF $<40 \%)$. Ejection fraction and wall motion abnormalities have also been detected during the follow-up (30 and 180 days after the initial hospitalization).

Transcranial color-coded duplex (TCCD) sonography was performed by two experienced investigators (BF, PS) using the $2 \mathrm{MHz}$ sector transducer of the GE Venue Go (GE Healthcare 9900 Innovation Drive Wauwatosa, WI 53226 U.S.A.) ultrasound device. He was unaware of the laboratory parameters and the other results, but knew that the patient suffered from SAH. The transtemporal window was insonated and orientation within the structure of the circle of Willis occurred in color mode. After identifying the different vessels of the circle, daily measurements of the middle cerebral, anterior cerebral and posterior cerebral arteries were performed on both sides. Regular TCCD measurements were registered in all patients between days 1 through 7. If ultrasound signs of vasospasm were present in any of the cases, duplex sonographies were performed until day 21 on a daily basis. Cerebral blood flow velocities, pulsatility indices and S/D ratios (systolic velocity/diastolic velocity) were documented in all cases. Based on previous suggestions, vasospasm was considered if mean blood flow velocity was higher than $120 \mathrm{~cm} / \mathrm{s}$ and severe vasospasm was diagnosed if mean blood flow velocity exceeded $200 \mathrm{~cm} / \mathrm{s}$ (19).

\section{Laboratory tests}

Creatine kinase activity was determined by a UV kinetic assay utilizing hexokinase and glucose- 6 phosphate dehydrogenase enzymes after the rate limiting CK step. CK-MB was expressed as CK-MB activity by using immunoinhibition with antibodies

to the CK-M subunit and subsequently measuring the CK activity as described above by using Roche reagents.

The high sensitive Troponin T assay was measured by an electrochemiluminescent (ECLIA) immunoassay on a Cobas e411 analyzer.

An ECLIA immunoassay was used measuring the N-terminal portion of the pro B-type natriuretic peptide (NT-pro-BNP) that has a considerably longer half life than the active peptide and thus is more suitable for risk stratification. 
Metanephrines are usually present in the urine in the form of glucuronate- or sulphate-conjugates, thus as the first step an acid hydrolysis was done. Subsequently, urine samples were diluted by a neutralizing buffer and the

measurement was carried out by an isocratic HPLC using an electrochemical detector (ABL and E-Jasco system).

\section{Statistical Analysis}

To analyse $r \times c$ contingency tables, we used $\chi 2$-tests. When $2 \times 2$ tables were analysed, we applied Yates' correction for continuity. When the number of expected cases was too small $(<5)$ in more than $20 \%$ of the cells, we used a $\times 2$-test with a simulated p-value calculated in a Monte-Carlo randomisation based on 2000 replicates as implemented in the 'chisq.test' function of $R$.

For the analysis of continuous variables, we checked the homogeneity of variances with the Bartlett test and checked the normal distribution of variables by the Shapiro-Wilks test. In the analysis of variables that were non-normally distributed and/or had heterogeneous variances, we used non-parametric statistical tests. In such cases, we applied Kruskal-Wallis tests, i.e., one-way non-parametric ANOVAs based on ranks to analyse differences between the study groups.

To analyse the effect of one or more continuous independent variables such as age on binary dependent variables such as the occurrence of TTC (no/yes), we applied binary logistic regression using the ' $\mathrm{glm}$ ' function of R and specifying binomial error distribution. To analyse the effect of one or more continuous independent variables on ordinal variables (that have qualitative levels that can be ordered), such as the severity of TTC (no TTC, mild and severe TTC), we applied ordinal logistic regression using the 'polr' function of R. When there were more than one independent variables, we first ran the full logistic regression model with all variables, and then removed non-significant $(p<0.05)$ variables in a backward stepwise fashion. We report $z$ values (binomial regression), $t$ values (ordinal regression) and odds ratios for logistic regression tests.

To analyse the effect of study group and continuous independent variables on continuous dependent variables, we applied general linear models (GLM) using the 'Im' function of R. For such tests, we report contrasts with the control group as reference (for study group) and slope coefficients \pm standard errors (for continuous independent variables) and $F$ values along with $p$ values.

For all statistical analyses, we used the R statistical environment, version 3.6.3. (R Core Team 2020). For all graphs, we used the 'ggplot2' package of R.

\section{Results}

One hundred thirty six patients were included to the study. The flowchart in Fig. 1 shows the study cohort, inclusion and exclusion, as well as the numbers of patients during follow-up. General patient characteristics and confounding factors are summarized in Table 1. The most important characteristics 
of subarachnoid hemorrhage in the entire cohort is shown in Table 2. In the entire cohort endovascular treatment was performed in 73, surgical clipping in 21 patients, in 42 patients no intervention was performed. A placement of an intraventricular drain was necessary in 40 cases, whereas lumbar drain was placed in 5 cases.

Table 1

General characteristics and confounding factors in the entire cohort

\begin{tabular}{|ll|}
\hline Parameter & Value \\
\hline Age (years) & $76.2(48-72)$ \\
\hline Gender (F/M) & $80 / 56$ \\
\hline Hypertension (Yes/No) & $73 / 63$ \\
\hline Arrhytmias or conduction disturbances (Yes/No) & $4 / 132$ \\
\hline Hypercholesterinemia/Trigliceridaemia (Yes/No) & $15 / 121$ \\
\hline Diabetes mellitus (Yes/No) & $5 / 131$ \\
\hline Hypothyreosis (Yes/No) & $3 / 133$ \\
\hline Hyperthyreosis (Yes/No) & $1 / 132$ \\
\hline Smoking (Yes/No) & $66 / 70$ \\
\hline Obesity (Yes/No) & $33 / 103$ \\
\hline
\end{tabular}


Table 2

Characteristics of subarachnoid hemorrhage in the entire cohort

\begin{tabular}{|c|c|}
\hline \multicolumn{2}{|l|}{ Location of the aneurysm $¥$} \\
\hline Internal carotid & 20 \\
\hline Anterior and middle cerebral & 30 \\
\hline Anterior and posterior communicating & 47 \\
\hline Other & 17 \\
\hline No aneurysm & 22 \\
\hline Ventricular bleeding (Yes/No) & $69 / 83$ \\
\hline Parenchymal bleeding (Yes/No) & $27 / 109$ \\
\hline \multicolumn{2}{|l|}{ Bleeding extent (side) } \\
\hline Left & 11 \\
\hline Right & 14 \\
\hline Bilateral & 2 \\
\hline No bleeding & 109 \\
\hline \multicolumn{2}{|l|}{ Bleeding location (lobe) } \\
\hline Frontal & 10 \\
\hline Frontotemporal & 3 \\
\hline Temporal & 7 \\
\hline Temporoparietal & 7 \\
\hline None & 109 \\
\hline \multicolumn{2}{|l|}{ Modified Fisher score at admission } \\
\hline 1 & 31 \\
\hline 2 & 19 \\
\hline 3 & 27 \\
\hline 4 & 59 \\
\hline \multicolumn{2}{|l|}{ Hunt-Hess grade at admission } \\
\hline 1 & 23 \\
\hline 2 & 37 \\
\hline
\end{tabular}




\begin{tabular}{|ll|}
\hline Location of the aneurysm $¥$ \\
\hline 3 & 31 \\
\hline 4 & 21 \\
\hline 5 & 24 \\
\hline WFNS score at admission & \\
\hline 1 & 62 \\
\hline 2 & 18 \\
\hline 3 & 7 \\
\hline 4 & 26 \\
\hline 5 & 23 \\
\hline GCS at admission & \\
\hline $3-4$ & 11 \\
\hline $5-6$ & 12 \\
\hline $7-8$ & 16 \\
\hline $9-12$ & 10 \\
\hline $13-14$ & 25 \\
\hline 15 & 62 \\
\hline
\end{tabular}

The incidence of TTC in the studied cohort

Takotsubo cardiomyopathy developed in 39 of the 136 patients $(28.7 \%)$. TTC was mild or severe in 28 (20.6\%) and $11(8.1 \%)$ patients, respectively.

The distribution of the different patterns according to the recommendation (2) was as follows: classical pattern: $n=10$; mid-ventricular pattern: $n=1$; reverse pattern: $n=4$; focal type: $n=21$; atypical pattern (global): $\mathrm{n}=3$. TTC developed within 7 days after admission in 4 cases, all were focal type.

The effect of age and gender on the occurence and severity of TTC

The occurrence of TTC was not related to the age of the patients (binomial logistic regression, odds ratio: $1.015, z=0.821, p=0.412$ ). Similarly, the severity of TTC was not related to patient age (ordinal logistic regression, coefficient $=0.013 \pm$ S.E. 0.018 , odds ratio: $1.013, t=0.729, p=0.466$ ).

Takotsubo cardiomyopathy was more frequent in females $(30 / 39 ; 77 \%)$ than in males $(9 / 39 ; 23 \%)$. TTC occurred in $30(37.5 \%)$ of the 80 female patients and in $9(16 \%)$ out of the 56 male patients. The 
difference was statistically significant (Yates-corrected $\chi 2=6.385, \mathrm{df}=1, \mathrm{p}=0.012$. TTC appeared to be more severe in female patients: $23(29 \%)$ presented with mild and $7(9 \%)$ with severe TTC, compared to male patients: $5(9 \%)$ mild and $4(7 \%)$ severe TTC cases, respectively $(\chi 2=8.152$, simulated $p=0.015)$.

The effect of comorbidities on the occurrence of TTC

The occurrence of TTC was not influenced by any of the comorbidities studied (Supplemental Table 1). A binomial logistic regression calculated in a general linear model testing the effect of each additional illness on the occurrence of TTC (yes/no) found that none of the eight illnesses/conditions influenced the occurrence of TTC (data not shown).

The relationship between comorbidities and severity of TTC

The severity of TTC was not influenced by any of the comorbidities studied (Supplemental Table 2). An ordinal logistic regression calculated in a general linear model testing the effect of each additional illness on the severity of TTC (none, mild, severe) found that none of the eight illnesses influenced the severity of TTC (data not shown).

The relationship between the occurrence of TTC and the severity scores at admission

The incidence of TTC was related to the modified Fisher scores; it increased from $13 \%$ in patients with Fisher score of 1 to $16 \%$ in score $2,30 \%$ in score $3 ; 41 \%$ in patients with score 4 (Table 3 ). The occurrence of TTC also increased with WFNS score from $18 \%$ in patients with score 1 and $14 \%$ in score 3 patients to $39 \%$ in score $2,35 \%$ in score 4 and $48 \%$ in score 5 patients (Table 3 ). None of the other variables influenced the occurrence of TTC (Table 3 ). 
Table 3

Occurrence of TTC in SAV variable categories. Significant variables are highlighted in Bold.

\begin{tabular}{|c|c|c|c|}
\hline SAV variable & No TTC & TTC & $\chi^{2}$ \\
\hline \multicolumn{4}{|l|}{ Location of aneurysm $¥$} \\
\hline Internal carotid & 12 & 8 & \\
\hline Anterior and middle cerebral & 24 & 6 & 2.984 n.s. \\
\hline Anterior and posterior communicating & 32 & 15 & \\
\hline Other & 12 & 5 & \\
\hline No aneurysm & 17 & 5 & \\
\hline Ventricular bleeding $(\mathrm{Y} / \mathrm{N})$ & $46 / 51$ & $23 / 16$ & 1.059 n.s. \\
\hline Parenchymal bleeding $(\mathrm{Y} / \mathrm{N})$ & $21 / 76$ & $6 / 33$ & 0.349 n.s. \\
\hline \multicolumn{4}{|l|}{ Bleeding extent (side) } \\
\hline Left & 8 & 3 & 1.311 n.s. \\
\hline Right & 11 & 3 & \\
\hline Bilateral & 2 & 0 & \\
\hline No bleeding & 76 & 33 & \\
\hline \multicolumn{4}{|l|}{ Bleeding location (lobe) } \\
\hline Frontal & 7 & 3 & 2.060 n.s. \\
\hline Frontotemporal & 3 & 0 & \\
\hline None & 76 & 33 & \\
\hline Temporal & 5 & 2 & \\
\hline Temporoparietal & 6 & 1 & \\
\hline \multicolumn{4}{|l|}{ Hunt-Hess grade } \\
\hline 1 & 18 & 5 & 6.856 n.s. \\
\hline 2 & 31 & 6 & \\
\hline 3 & 19 & 12 & \\
\hline 4 & 15 & 6 & \\
\hline
\end{tabular}

Note: ${ }^{\star} p<0.05 ;$ n.s. indicates non-significant difference

‡ neighbouring values or similar categories were pooled to meet $\chi^{2}$-test assumptions 


\begin{tabular}{|c|c|c|c|}
\hline SAV variable & No TTC & TTC & $\chi^{2}$ \\
\hline 5 & 14 & 10 & \\
\hline \multicolumn{4}{|c|}{ Modified Fisher score } \\
\hline 1 & 27 & 4 & 9.481 * \\
\hline 2 & 16 & 3 & $p=0.024$ \\
\hline 3 & 19 & 8 & \\
\hline 4 & 35 & 24 & \\
\hline \multicolumn{4}{|l|}{ WFNS } \\
\hline 1 & 51 & 11 & 9.823 * \\
\hline 2 & 11 & 7 & $p=0.041$ \\
\hline 3 & 6 & 1 & \\
\hline 4 & 17 & 9 & \\
\hline 5 & 12 & 11 & \\
\hline \multicolumn{4}{|l|}{ GCS } \\
\hline $3-4$ & 6 & 5 & \\
\hline $5-6$ & 6 & 6 & \\
\hline $7-8$ & 13 & 3 & 13.509 * \\
\hline $9-12$ & 4 & 6 & $p<0.05$ \\
\hline $13-14$ & 17 & 8 & \\
\hline 15 & 51 & 11 & \\
\hline \multicolumn{4}{|c|}{ Note: ${ }^{*} p<0.05 ; n . s$. indicates non-significant difference } \\
\hline
\end{tabular}

Relationship between severity of TTC and severity scores at admission

The severity of TTC was related to modified Fisher score, Hunt-Hess score, WFNS score and GCS (Table 4). The incidence of mild TTC was $13 \%, 11 \%, 26 \%$ and $25 \%$ in patients with modified Fisher score of 1, 2, 3 and 4, respectively, whereas the incidence of severe TTC increased from 0 in patients with score 1 to $5 \%$ in score $2,4 \%$ in score 3 and $15 \%$ in score 4 patients (Table 4 ). 
Table 4

Severity of TTC in different SAH variable categories. Significant effects are highlighted in Bold.

\begin{tabular}{|c|c|c|c|c|}
\hline SAV variable & $\begin{array}{l}\text { No TTC } \\
(n=97)\end{array}$ & $\begin{array}{l}\text { Mild TTC } \\
(n=28)\end{array}$ & $\begin{array}{l}\text { Severe TTC } \\
(n=11)\end{array}$ & $\chi^{2}$ \\
\hline \multicolumn{4}{|l|}{ Location of aneurysm $\neq$} & \multirow[t]{5}{*}{3.836 n.s. } \\
\hline Internal carotid & 12 & 5 & 3 & \\
\hline Middle and anterior cerebral & 24 & 4 & 2 & \\
\hline Anterior and posterior communicating & 32 & 11 & 4 & \\
\hline Other & 12 & 4 & 1 & \\
\hline No aneurysm & 17 & 4 & 1 & \\
\hline Ventricular bleeding $(\mathrm{Y} / \mathrm{N})$ & $46 / 51$ & $14 / 14$ & $9 / 2$ & 4.684 n.s. \\
\hline Parenchymal bleeding $(\mathrm{Y} / \mathrm{N})$ & $21 / 76$ & $4 / 24$ & $2 / 9$ & 0.761 n.s. \\
\hline \multicolumn{4}{|l|}{ Bleeding side } & \multirow[t]{5}{*}{1.392 n.s. } \\
\hline Left & 8 & 2 & 1 & \\
\hline Right & 11 & 2 & 1 & \\
\hline Bilateral & 2 & 0 & 0 & \\
\hline No bleeding & 76 & 24 & 9 & \\
\hline \multicolumn{5}{|l|}{ Bleeding extent (lobe) } \\
\hline Frontal & 7 & 2 & 1 & \multirow[t]{5}{*}{2.779 n.s. } \\
\hline Frontotemporal & 3 & 0 & 0 & \\
\hline None & 76 & 24 & 9 & \\
\hline Temporal & 5 & 1 & 1 & \\
\hline Temporoparietal & 6 & 1 & 0 & \\
\hline \multicolumn{4}{|l|}{ Hunt-Hess grade } & \multirow{4}{*}{$\begin{array}{l}22.687 \text { ** } \\
p=0.004\end{array}$} \\
\hline 1 & 18 & 5 & 0 & \\
\hline 2 & 31 & 6 & 0 & \\
\hline 3 & 19 & 9 & 3 & \\
\hline \multicolumn{5}{|l|}{ Note: ${ }^{\star} p<0.05 ; * \star p<0.01 ; * \star \star p<0.001$} \\
\hline$\ddagger$ neighbouring values or similar catec & s were & oled to $n$ & $2-t$ & ons \\
\hline
\end{tabular}




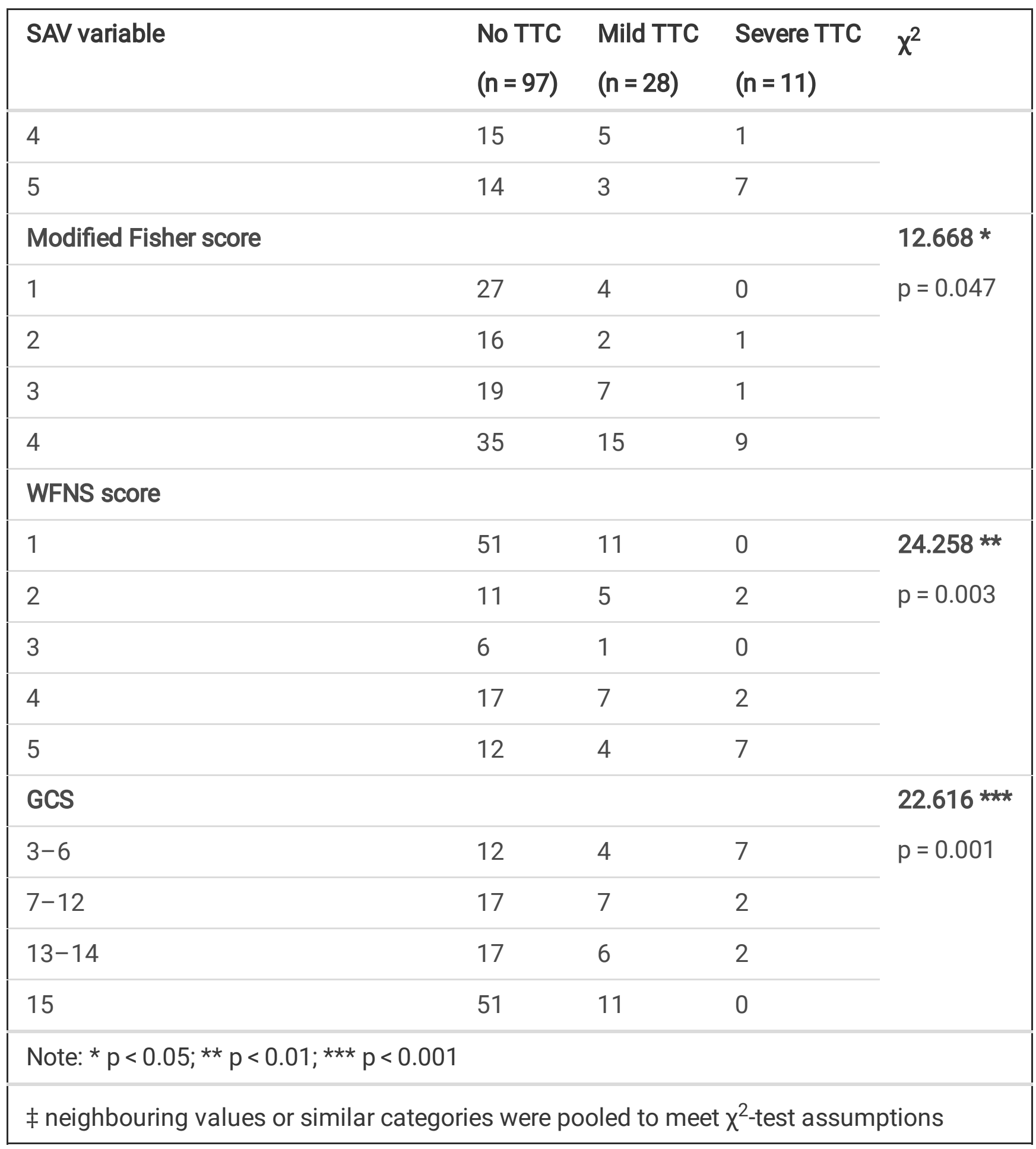

The incidence of mild TTC varied between $13 \%$ (Hunt-Hess score 5) and $29 \%(\mathrm{H}-\mathrm{H}$ score 3 ) with no obvious trend, whereas the incidence of severe TTC increased from 0 in patients with scores 1 and 2 to $10 \%$ in score $3,5 \%$ in score 4 and $29 \%$ in score 5 patients (Table 4). The incidence of mild TTC also varied between $17 \%$ and $28 \%$ in the five WFNS score groups, while the incidence of severe TTC increased from 0 in scores 1 and 3 to $11 \%$ in score $2,8 \%$ in score 4 and $30 \%$ in score 5 (Table 4). Finally, the incidence of severe TTC was highest (30\%) in GCS scores 3 to 6 , was lower in scores 7 to 12 and 13 to 14 (8\% each) was 0 in score 15; mild TTC varied in frequency between 17 and $27 \%$ with no obvious trend (Table 4). 
When the effects of all SAV variables were tested simultaneously in a binomial logistic regression followed by backward stepwise removal of non-significant effects, only the modified Fisher score influenced the incidence of TTC, and a positive coefficient indicated that TTC was more likely to occur in patients with higher modified Fisher scores (estimate: $0.54 \pm$ S.E. 0.185 , odds ratio: $1.719, z=2.923, p=$ 0.003). Similarly, the severity of TTC showed a positive relationship with only the modified Fisher score (estimate: $0.57 \pm 0.185$, odds ratio: $1.764, z=3.071, p=0.036$ ).

Relationship between development of vasospasm and TTC:

Vasospasm (any type) occurred in 23/97 patients in the control (non TTC) group and 13/39 in the TTC group (Chi2:0.74; $P=0.36$ ), indicating that TS was not more frequent in SAH patients in whom vasospasm developed. Severe vasospasm occurred in 5/97 patients and 6/39 patients in the non-TTC and TTC groups, respectively (Chi2:2,87; $p=0.09$.)

\section{Laboratory parameters}

For CK, the differences between the three groups were significant on day 1 (Kruskal-Wallis $\chi 2=11.642$, df $=2, p=0.003)$, day $2(x 2=8.673, d f=2, p=0.013)$ and day $4(x 2=7.478, d f=2, p=0.024)$ but not on the other days. For CK-MB, the differences were significant on day $1(x 2=9.346, d f=2, p=0.009)$, but not on the other days. For CTNT, the differences were highly significant each day, mostly because of high values in the severe TTC group (Fig. 2).

For NTPBNP, the differences were also highly significant each day because of high values in the severe TTC group (Fig. 3)

General linear models showed that the level of metanephrin in the urine did not differ between the three study groups and was not influenced by the level of arterenol or dobutamin administered, and this was true for the day of admission, as well as days 30 and 180 of follow up (results not shown). In contrast, the level of normetanephrin differed between TTC groups and was influenced by arterenol and dobutamin administration (Fig. 4, Supplemental Table 3). The interaction between groups and arterenol or dobutamin were not significant (results not shown), indicating that the effects of the drugs were similar in each group. The difference between study groups was marginally non-significant 30 days after the bleeding, partly due to a slight difference between the control and the mild TTC group and partly due to few data in the severe TTC group (Fig. 4B, Supplemental Table 3). Finally, there was no difference in normetanephrin concentration between the study groups 180 days after the bleeding $(G L M, F 2,54=0.209$, $p=0.812)$.

Changing the echocardiography parameters over time

The ejection fraction was significantly lower in patients with severe TTC than in non-TTC controls, and was intermediate but closer to level of controls in patients in patients with mild on both day 1 and 7 (Fig. 5A-D). These differences were marginally significant on day 30 and disappeared by day 180 . Thus, 
ejection fraction improved stepwise in TTC patients during the follow-up period, but there were still signs of weak contractile function.

The wall motion score index was highest in patients in the severe TTC group, and low in the mild TTC and control groups (Fig. 5E-H). The differences were significant on all study days, indicating that the difference could be detected even after 180 days.

Relationship between severity of TTC and mortality

By the end of the follow-up period (180 days), 70 (74.5\%) patients survived in the control (non-TTC) group $(n=94), 22(81.5 \%)$ survived in the mild TTC group $(n=27)$ and three $(27 \%)$ survived in the severe TTC group $(n=11)$. The difference in mortality rate was significant $(X 2=12.395, d f=2, p=0.002)$. The number of deceased patients during different phases of follow up were as follows:

- Days 1-7: NoTTC group: $n=9$; mild TTC: $n=2$, severe TTC: $n=6$

- Days 8-30: No TTC: $\mathrm{n}=6$; mild TTC: $\mathrm{n}=3$; severe TTC: $\mathrm{n}=1$

- Days 31-180: No TTC: $n=9$; mild TTC: $n=0$; severe TTC: $n=1$

Relationship between the severity of TTC and the outcome of the patients

GOS, Barthel, and Karnofsky outcome scores were higher in patients in the control (non-TTC) and the mild TTC groups than in the severe TTC group (Fig. 6.). All the differences were significant, with the exception of the differences in Barthel scores during the second check-up (Fig. 6E), indicating that severe TTC is associated with worse long-term clinical outcome of the patients.

\section{Discussion}

In this prospective follow-up study of non-traumatic subarachnoid hemorrhage patients we found that cardiac wall motion disturbances can be detected in $28.7 \%$ of the cases. TTC may influence long-term mortality, and severe TTC may affect long term outcome.

The prevalence of TTC varied in a wide range in previous reports, reaching up to $30 \%$ (5). The differences could be, at least in part explained by developments in the definition of TTC over time: typical classical form of TTC was found in $2.2 \%$ of SAH patients in the study of Talahma (20), while others reported on $8.8 \%$ (9), $11 \%$ (10), 22\% (21) and 27\% (11) wall motion abnormalities. The reported incidence may have been influenced by the nature of the study (prospective or retrospective) and the timing and frequency of echocardiographic assessments. The prevalence of TTC in SAH may be underestimated by the majority of the studies (22) and prospective studies using regular echocardiography follow-up have been encouraged (15). In the present study we included only patients in whom transthoracic echocardiography was performed within 24 hours after admission, and ECG and cardiac biomarkers were regularly checked on the daily basis. Whenever a significant ECG change was found, echocardiography was performed immediately, in addition to the day 1 and day 7 examinations. Therefore, we believe that the $28.7 \%$ 
incidence rate of TTC in our cohort reflects the true incidence of this condition; all cases of newly appearing wall motion abnormality were included. During the off-line analysis, we arbitrarily defined a mild and a severe group of TTC patients based on the ejection fraction; the predefined threshold of $40 \%$ was based on clinical experience that showed that below this level the cardiovascular status of the patients usually worsens and pharmacologic intervention is often necessary. Based on this grouping, we found the incidence of severe TTC $8.1 \%$ in SAH, which corresponds to the prevalence rates that have been published previously (9).

In line with previous studies we also found that TTC in our cohort was more frequent in females than in males, and more severe cases were found among women. In two retrospective studies, the percentage of females were $100 \%$ (20) and $78 \%$ (5), respectively. In a prospective study of Kothavale et al. (22) the represantation of females was $68 \%$. To explain these differences it is proposed that estrogen deficiency in postmenopausal women results in an increased sympathetic drive and endothelial dysfunction, making cardiac microcirculation more vulnerable during critical illnesses (23).

In contrast to previous reports, we could not find any relationship between occurrence of TTC and any of the cardiovascular risk factors and comorbidities. This may be expained by a relatively high prevalence of the cardiovascular risk factors in the Hungarian general population.

We found that the incidence of TTC was associated with modified Fisher scores and WFNS scores. Moreover, the severity of TTC was related to higher modified Fisher scores, Hunt\&Hess scores, WFNS scores and GCS. This indicates that the more severe is the SAH at admission, the more severe TTC develops during the course of the disease. Similar observations were published by Talahma et al. (20). Malik et al. also found a positive association between Hunt\&Hess scores and left ventricular ejection fraction (24). Kothavalet et al. (22) and Kilbourn et al. (25) found that Hunt\&Hess grade 3-5 has a predictive value for the presence of regional wall motion abnormalities. It has to be noted, that in the present study binominal regression testing revealed that of the severity scores, only the modified Fisher score on admission was strongly associated with severity of TTC.

Follow-up investigations of cardiac biomarkers showed that both cardiac troponin and NT-proBNP values were elevated from the day of admission both in the mild and the severe TTC groups. While troponin T and NT-proBNP levels tended to normalize in the mild group from day 3 on, in the severe group higher levels of these cardiac biomarkers persisted by day 7. The time pattern of the elevation of cardiac troponin is in line with previous observations $(24,26)$ confirming its high sensivity to detect left ventricular dysfunction in SAH. Although elevated serum BNP was significantly associated with regional wall motion abnormalities, it was not associated with 30 - day mortality in previous studies $(27,28)$.

It is widely accepted that catecholamines play a key role in the development of TTC. Elevation of plasma catecholamine (epinephrine and norepinephrine) concentrations are typical findings in patients with TTC $(29-31)$. At rest, the source of norepinephrine (NE) acting on the cardiac receptors is the andrenal medulla in $2-8 \%$ percent, and the rest is released by the sympathetic nerve endings (32). In the acute phase of TTS, an increased concentration of circulating catecholamines, and an increased production of 
catecholamines at the myocardial level has been observed. Further, the density of the beta-2 receptors is higher in the apical than in the basal part of the ventricle, whereas beta1-adrenergic receptors are expressed predominantly at the base. A physiologic rise in the levels of epinephrine results in a switch of beta2-adrenoceptor coupling from $\mathrm{Gs}$ to $\mathrm{Gi}$, in order to prevent the proapoptotic effect of the intense stimulation of the beta- 1 adrenoceptors. Additionally, switching of the coupling mechanism leads to a negative inotropic effect on the apex of the left ventricle $(33,34)$. Additionally, increased cardiac sympathetic activity may cause endothelial dysfunction and consequent coronary microvascular constriction, thus contributing to myocardial ischemia (35). In accordance with these, in the present study we found that metanephrine concentration in the urine did not differ between TTC and non-TTC patients, whereas normetanephrine was elevated in patients with TTC at admission. This difference was missing at day 30 and day 180 of the follow up. Normetanephrine concentrations were not influenced by dobutamine or norepinephrine treatment. In line with these observations, Akashi et al. demonstrated an improvement of cardiac sympathetic hyperactivity in TCC patients using myocardial scintigraphy (36). It is tempting to hypothetise that severe TTC itself, via the systemic effects of myocardial dysfunction, may contribute to the elevation of normetanephrin production and worsening of TTC as part of a vicious circle. However, our data are insufficient to confirm this.

Per definition, wall motion abnormalities are temporary in patients with TTC and show and improvement over time along with the ejection fraction of the heart. Kim and co-workers demonstrated an improvement of the ejection fraction from $38 \%$, to $61 \%$ during a 6 week follow-up (37). In a study of Templin et al. reduced left ventricular ejection fraction (mean value: 40.7 ) was found in $86.5 \%$ of patients with TTC, which showed a recovery over time during a 60-day follow-up (38). Similarly, Dias et el. reported on an improvement of the left ventricular ejection fraction from the intial $32.1-54 \%$ in a 6-month follow up study. (39). The EF tended to normalize in mild cases, while in the severe TTC group low EF persisted at 7 days. Although at 30 days statistically significant differences between the TTC groups compared to nonTTC groups could not be demonstrated, it has to be noted that EF was still lower in severe cases and the lack of statistical difference might be explaned by the relatively small sample size. Importantly, wall motion score index differences persisted over 180 days in the severe TTC group, indicating that the contractile function of the heart may not entirely recover at 6 months, despite normalization of the global left ventricular ejection fraction.

The severity of TTC in our cohort significantly affected the outcome and mortality of the patients during the 180 days follow-up. The mortality at 6 months was significantly higher among patients with severe TTC whereas the mortality in mild TTC cases was comparable with that of non-TTC subarachnoid hemorrhage patients. The mortality of the present study (33.3\%) is lower than that in the study of Kilbourn (47\%) (25), buth slightly higher that reported by Talahma (28\%) (20) and Abd (26\%) (40). In those who were alive at the day 180 follow-up, both Glasgow outcome scores and Karnovsky scores were significantly lower in the severe compared to the mild TTC group. Barthel's index reflected worse outcome in the severe TTC group only at 30 days in the present study. There are only few studies that have assessed long-term outcome of TTC. Similarly to our results, Crago et al. demonstrated differences in Barthel's index at 30 days but not at 60 days in TCC patients after subarachnoid hemorrhage (41). Mutoh 
et al. reported on poor 3-month functional outcome as assessed by modified Rankin Scale in TTC patients with an $\mathrm{EF}<40 \%$ (42).

We have to mention some limitations of our study. This was a single center follow-up study with an inclusion period of 22 months and as a consequence, the number of included patients is limited. We may also be criticized for the follow-up of vasospasm with transcranial color-coded duplex sonography. In fact, the vasospasm may be accurately diagnosed by angiography, but we decided to perform a regular TCCD check of the blood flow velocities. If clinical and/or ultrasonographic signs of vasospasm were found, cerebral angiography was performed. We did not measure the blood levels of epinephrine and norepinephrine because we belive that sampling blood for these parameters would represent a rapidly changing snapshot, while urine metanephrine and normetanephrine in 24 hour collected urine may be more representative of the tissue exposure to these hormones. Finally, we arbitrarily defined mild and severe forms of TTC during analysis of the results based on ejection fraction. The rationale behind this dichotomization was that previous reports indicated that the critical threshold of ejection fraction that determines cardiovascular complications and long-term outcome lies between 40-45\% (38).

\section{Conclusions}

We found that Takotsubo cardiomyopathy is a frequent phenomenon occurring in patients with aneurysmal subrachnoid hemorrhage, and severe TTC is present in $8 \%$ of SAH cases. The incidence of SAH is associated with the modified Fisher and WFNS scores at admission. The severity of TTC may may be an independent predictor of mortality and outcome at 6 months after disease onset. Systematic and regular follow-up of ECG and echocardiographic abnormalities is warranted in patients with subrachnoid hemorrhage for early detection of TTC. Further studies are required to identify the best treatment approach to the cardiac changes which may accompany SAH.

\section{Abbreviations}

TTC

takotsubo cardiomyopathy

SAH

subarachnoid hemorrhage

ICU

intensive care unit

LV

left ventricle

RV

right ventricle

EF

ejection fraction

GCS 
Glasgow Coma Score

WFNS

World Federation of Neurological Society

ECG

electrocardiogram

CT

computed tomography

TCCD

Transcranial color-coded duplex

\section{Declarations}

- Ethics approval and consent to participate:The protocol was approved by the Institutional Ethics Committee of the University of Debrecen (registration number: DE RKEB/IKEB:4317-2015). All included patients or their closest relatives gave written informed consent before participation.

- Consent for publication: All authors read the final version of the manuscript and gave consent to submission.

- Availability of data and materials: all available material (including raw data and statistical analysis will be made available upon request from the corresponding author.

- Competing interests. the authors declare that they do not have any competing interest to report

- Funding: Hungarian Brain research program [grant number KTIA_13_NAP-A-II/5], the founder is the Hungarian Academy of Sciences

- Authors' contributions: CM: conceptualisation, writing the manuscript, patient care; JG: patient care; drafting the mansucript; DS: patient care, handling of data; LF: echocardiography, cardiologic consultation; AS: echocardiography, cardiologic consultation; PS: patient care, transcranial Doppler; EVN: endocrinology consultation, drafting the manuscript; SL: statistical analysis; JK: laboratory analysis consultation, drafting the manuscript; BF: conceptualisation, drafting the manuscript, transcranial Doppler measurements, patient care

- Acknowledgements: none

- Authors' information (optional)

\section{References}

1. Dote K, Sato H, Tateishi H, Uchida T, Ishihara M. Myocardial stunning due to simultaneous multivessel coronary spasms: a review of 5 cases [in Japanese]. J Cardiol. 1991;21:203-14.

2. Dias A, Núñez Gil IJ, Santoro F, Madias JE, Pelliccia F, Brunetti ND, Salmoirago-Blotcher E, Sharkey SW, Eitel I, Akashi YJ, El-Battrawy I, Franco E, Akin I, Jaguszewski M, Dawson D, Figueredo VM, Napp LC, Christensen TE, Hebert K, Ben-Dor I, Ozaki Y, García-Garcia HM, Kajita AH, Akasaka T, Kurisu S, 
Lerman A, Waksman R. Takotsubo syndrome: State-of-the-art review by an expert panel - Part 1. Cardiovasc Revasc Med. 2019;20:70-9.

3. Deshmukh A, Kumar G, Pant S, Rihal C, Murugiah K, Mehta JL. Prevalence of Takotsubo cardiomyopathy in the United States. Am Heart J. 2012;164:66-71.e1.

4. Bybee KA, Prasad A. Stress-related cardiomyopathy syndromes. Circulation. 2008;118:397-409.

5. Kerro A, Woods T, Chang JJ. Neurogenic stunned myocardium in subarachnoid hemorrhage. J Crit Care. 2017;38:27-34.

6. Dias V, Cabral S, Meireles A, Gomes C, Antunes N, Vieira M, et al. Stunned myocardium following ischemic stroke. Case report. Cardiology. 2009;113:287-90.

7. Shimizu M, Kagawa A, Takano T, Masai H, Miwa Y. Neurogenic stunned myocardium associated with status epileptics and postictal catecholamine surge. Intern Med. 2008;47:269-73.

8. Nasr DM, Tomasini S, Prasad A, Rabinstein AA. Acute Brain Diseases as Triggers for Stress Cardiomyopathy: Clinical Characteristics and Outcomes. Neurocrit Care. 2017;27:356-61.

9. Mayer SA, LiMandri G, Sherman D, Lennihan L, Fink ME, Solomon RA, DiTullio M, Klebanoff LM, Beckford AR, Homma S. Electrocardiographic markers of abnormal left ventricular wall motion in acute subarachnoid hemorrhage. J Neurosurg. 1995;83:889-96.

10. Temes RE, Tessitore E, Schmidt JM, Naidech AM, Fernandez A, Ostapkovich ND, Frontera JA, Wartenberg KE, Di Tullio MR, Badjatia N, Connolly ES, Mayer SA, Parra A. Left ventricular dysfunction and cerebral infarction from vasospasm after subarachnoid hemorrhage. Neurocrit Care. 2010;13:359-65.

11. Khush K, Kopelnik A, Tung P, Banki N, Dae M, Lawton M, Smith W, Drew B, Foster E, Zaroff J. Age and aneurysm position predict patterns of left ventricular dysfunction after subarachnoid hemorrhage. $J$ Am Soc Echocardiogr. 2005;18:168-74.

12. Zaroff JG, Rordorf GA, Ogilvy CS, Picard MH. Regional patterns of left ventricular systolic dysfunction after subarachnoid hemorrhage: evidence for neurally mediated cardiac injury. J Am Soc Echocardiogr. 2000;13:774-9.

13. Cinotti R, Piriou N, Launey Y, Le Tourneau T, Lamer M, Delater A, Trochu JN, Brisard L, Lakhal K, Bourcier R, Desal H, Seguin P, Mallédant Y, Blanloeil Y, Feuillet F, Asehnoune K, Rozec B. Speckle tracking analysis allows sensitive detection of stress cardiomyopathy in severe aneurysmal subarachnoid hemorrhage patients. Intensive Care Med. 2016;42:173-82.

14. Madias JE. Forme fruste cases of Takotsubo syndrome: a hypothesis. Eur J Intern Med. 2014;25:e47.

15. Madias JE. What is the real prevalence of Takotsubo syndrome in patients admitted with aneurysmal subarachnoid hemorrhage? Clin Neurol Neurosurg. 2016;145:104-5.

16. Diringer MN, Bleck TP, Claude Hemphill J 3rd, Menon D, Shutter L, Vespa P, Bruder N, Connolly ES Jr, Citerio G, Gress D, Hänggi D, Hoh BL, Lanzino G, Le Roux P, Rabinstein A, Schmutzhard E, Stocchetti N, Suarez JI, Treggiari M, Tseng MY, Vergouwen MD, Wolf S, Zipfel G; Neurocritical Care Society. Critical care management of patients following aneurysmal subarachnoid hemorrhage: 
recommendations from the Neurocritical Care Society's Multidisciplinary Consensus Conference. Neurocrit Care. 2011;15:211-240.

17. Prasad A, Lerman A, Rihal CS. Apical ballooning syndrome (tako-tsubo or stress cardiomyopathy): a mimic of acute myocardial infarction. Am Heart J. 2008;155:408-17.

18. Lebeau R, Serri K, Lorenzo MD, Sauvé C, Le VHV, Soulières V, El-Rayes M, Pagé M, Zaïani C, Garot J, Poulin F. Assessment of LVEF using a new 16-segment wall motion score in echocardiography. Echo Res Pract. 2018;5:63-9.

19. Lysakowski C, Walder B, Costanza MC, Tramèr MR. Transcranial Doppler versus angiography in patients with vasospasm due to a ruptured cerebral aneurysm: A systematic review. Stroke. 2001;32:2292-8.

20. Talahma M, Alkhachroum AM, Alyahya M, Manjila S, Xiong W. Takotsubo cardiomyopathy in aneurysmal subarachnoid hemorrhage: Institutional experience and literature review. Clin Neurol Neurosurg. 2016;141:65-70.

21. Davies KR, Gelb AW, Manninen PH, Boughner DR, Bisnaire D. Cardiac function in aneurysmal subarachnoid haemorrhage: a study of electrocardiographic and echocardiographic abnormalities. Br J Anaesth. 1991;67:58-63.

22. Kothavale A, Banki NM,Kopelnik A, Yarlagadda S. Lawton MT,Ko N,etal. Predictors of left ventricular regional wall motion abnormalities after subarachnoid hemorrhage. Neurocrit Care. 2006;4:199-205.

23. Pelliccia F, Kaski JC, Crea F, Camici PG. Pathophysiology of Takotsubo Syndrome. Circulation. 2017;135:2426-41.

24. Malik AN, Gross BA, Rosalind Lai PM, Moses ZB, Du R. Neurogenic stress cardiomyopathy after aneurysmal subarachnoid hemorrhage. World Neurosurg. 2015;83:880-5.

25. Kilbourn KJ, Levy S, Staff I, Kureshi I, McCullough L. Clinical characteristics and outcomes of neurogenic stress cadiomyopathy in aneurysmal subarachnoid hemorrhage. Clin Neurol Neurosurg. 2013;115:909-14.

26. Parekh N, Venkatesh B, Cross D, Leditschke A, Atherton J, Miles W, et al. Cardiac troponin I predicts myocardial dysfunction in aneurysmal subarachnoid hemorrhage. J Am Coll Cardiol. 2000;36:132835 .

27. Tung P, Kopelnik A, Banki N, Ong K, Ko N, Lawton MT, et al. Predictors of neurocardiogenic injury after subarachnoid hemorrhage. Stroke. 2004;35:548-51.

28. Duello KM, Nagel JP, Thomas CS, Blackshear JL, Freeman WD. Relationship of troponin T and ageand sex-adjusted BNP elevation following subarachnoid hemor- rhage with 30-day mortality. Neurocrit Care. 2015;23:59-65.

29. Song BG, Chun WJ, Park YH, Kang GH, Oh J, Lee SC, Park SW, Oh JK. The clinical characteristics, laboratory parameters, electrocardiographic, and echocardiographic findings of reverse or inverted takotsubo cardiomyopathy: comparison with mid or apical variant. Clin Cardiol. 2011;34:693-9.

30. Kume T, Akasaka T, Kawamoto T, Yoshitani H, Watanabe N, Neishi Y, et al. Assessment of coronary microcirculation in patients with takotsubo-like left ventricular dysfunction. Circ J. 2005;69(8):934-9. 
31. Wittstein IS, Thiemann DR, Lima JAC, Baughman KL, Schulman SP, Gersten-blith G, Wu KC, Rade JJ, Bivalacqua TJ, Champion HC. Neurohumoral fea-tures of myocardial stunning due to sudden emotional stress. N Engl J Med. 2005;352:539-48.

32. Janig W. The Integrative Action of the Autonomic Nervous System. Cambridge: Cambridge University Press; 2006.

33. Lyon AR, Bossone E, Schneider B, Sechtem U, Citro R, Underwood SR, Sheppard MN, Figtree GA, Parodi G, Akashi YJ, Ruschitzka F, Filippatos G, Mebazaa A, Omerovic E. Current state of knowledge on Takotsubo syndrome: a Position Statement from the Taskforce on Takotsubo Syndrome of the Heart Failure Association of the European Society of Cardiology. Eur J Heart Fail. 2016;18:8-27.

34. Lyon AR, Rees PS, Prasad S, Poole-Wilson PA, Harding SE. Stress (Takotsubo) cardiomyopathy-a novel pathophysiological hypothesis to explain catecholamine-induced acute myocardial stunning. Nat Clin Pract Cardiovasc Med. 2008;5:22-9.

35. Pelliccia F, Kaski JC, Crea F, Camici PG. Pathophysiology of Takotsubo Syndrome Circulation. 2017;135:2426-41.

36. Akashi YJ, Nakazawa K, Sakakibara M, Miyake F, Musha H, Sasaka K. 123I-MIBG myocardial scintigraphy in patients with "takotsubo" cardiomyopathy. J Nucl Med. 2004;45:1121-7.

37. Kim H, Senecal C, Lewis B, Prasad A, Rajiv G, Lerman LO, Lerman A. Natural history and predictors of mortality of patients with Takotsubo syndrome. Int J Cardiol. 2018;267:22-7.

38. Templin C, Ghadri JR, Diekmann J, Napp LC, Bataiosu DR, Jaguszewski M, Cammann VL, Sarcon A, Geyer V, Neumann CA, Seifert B, Hellermann J, Schwyzer M, Eisenhardt K, Jenewein J, Franke J, Katus HA, Burgdorf C, Schunkert H, Moeller C, Thiele H, Bauersachs J, Tschöpe C, Schultheiss HP, Laney CA, Rajan L, Michels G, Pfister R, Ukena C, Böhm M, Erbel R, Cuneo A, Kuck KH, Jacobshagen C, Hasenfuss G, Karakas M, Koenig W, Rottbauer W, Said SM, Braun-Dullaeus RC, Cuculi F, Banning A, Fischer TA, Vasankari T, Airaksinen KE, Fijalkowski M, Rynkiewicz A, Pawlak M, Opolski G, Dworakowski R, MacCarthy P, Kaiser C, Osswald S, Galiuto L, Crea F, Dichtl W, Franz WM, Empen K, Felix SB, Delmas C, Lairez O, Erne P, Bax JJ, Ford I, Ruschitzka F, Prasad A, Lüscher TF. Clinical Featuresand Outcomes of Takotsubo (Stress) Cardiomyopathy. N Engl J Med. 2015;373:929-938.

39. Dias A, Franco E, Rubio M, Bhalla V, Pressman GS, Amanullah S, Hebert K, Figueredo VM. Usefulness of left ventricular strain analysis in patients with takotsubo syndrome during acute phase. Echocardiography. 2018;35:179-83.

40. Abd TT, Hayek S, Cheng JW, Samuels OB, Wittstein IS, Lerakis S. Incidence and clinical characteristics of takotsubo cardiomyopathy post-aneurysmal subarachnoid hemorrhage. Int $J$ Cardiol. 2014;176:1362-4.

41. Crago EA, Kerr ME, Kong Y, Baldisseri M, Horowitz $M$, Yonas $H$, Kassam A. The impact of cardiac complications on outcome in the SAH population. Acta Neurol Scand. 2004;110(4):248-53.

42. Mutoh T, Kazumata K, Terasaka S, Taki Y, Suzuki A, Ishikawa T. Impact of transpulmonary thermodilution-based cardiac contractility and extravascular lung water measurements on clinical 
outcome of patients with Takotsubo cardiomyopathy after subarachnoid hemorrhage: a retrospective observational study. Crit Care. 2014;18:482.

\section{Figures}

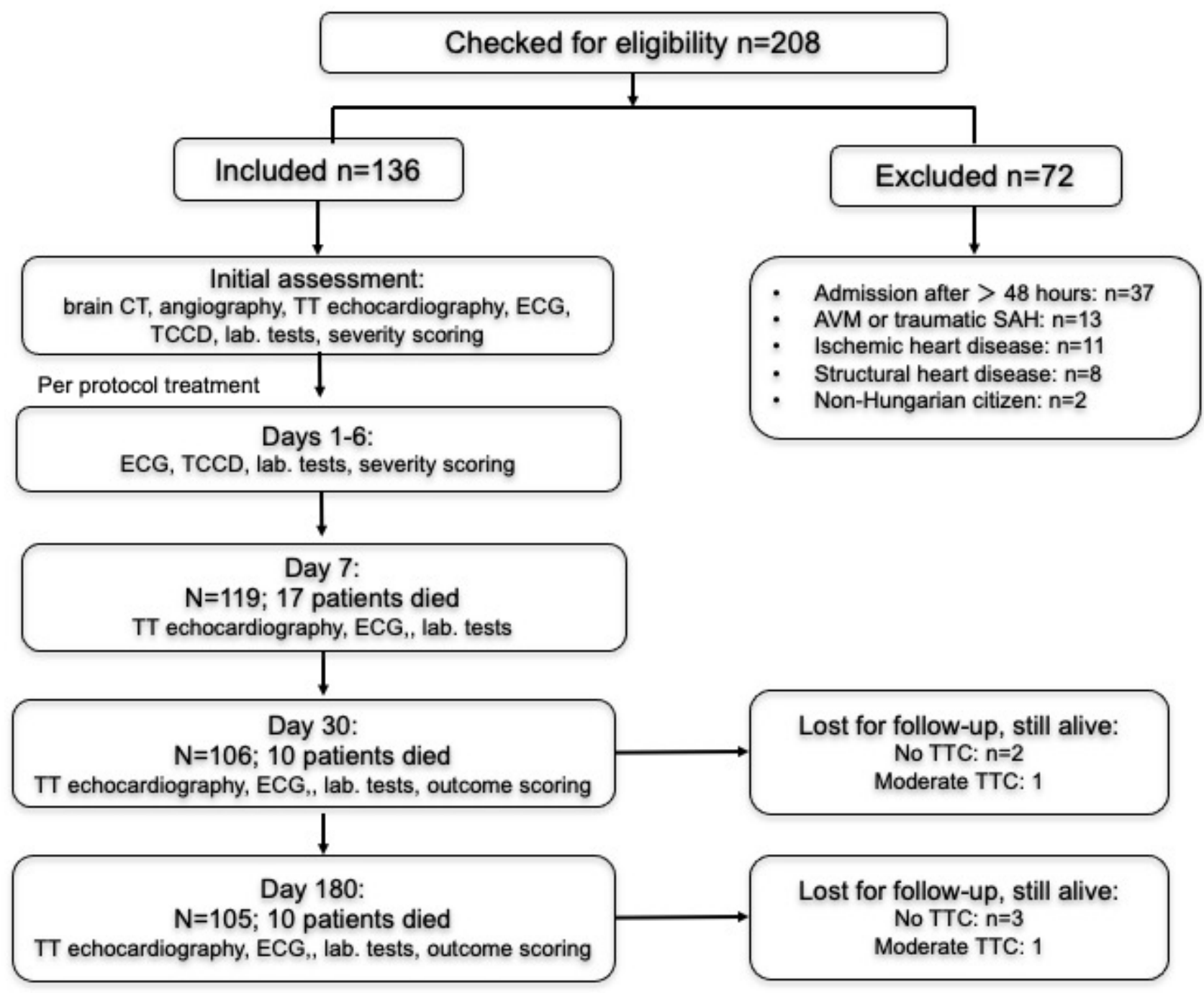

\section{Figure 1}

Flowchart of patient inclusion and exclusion. CT indicates computed tomography; TT indicates transthoracic; ECG indicates electrocardiogramm; TCCD indicates transcranial color-coded duplex sonography 
Takotsubo study group: 追 Control 官 Moderate 追 Severe

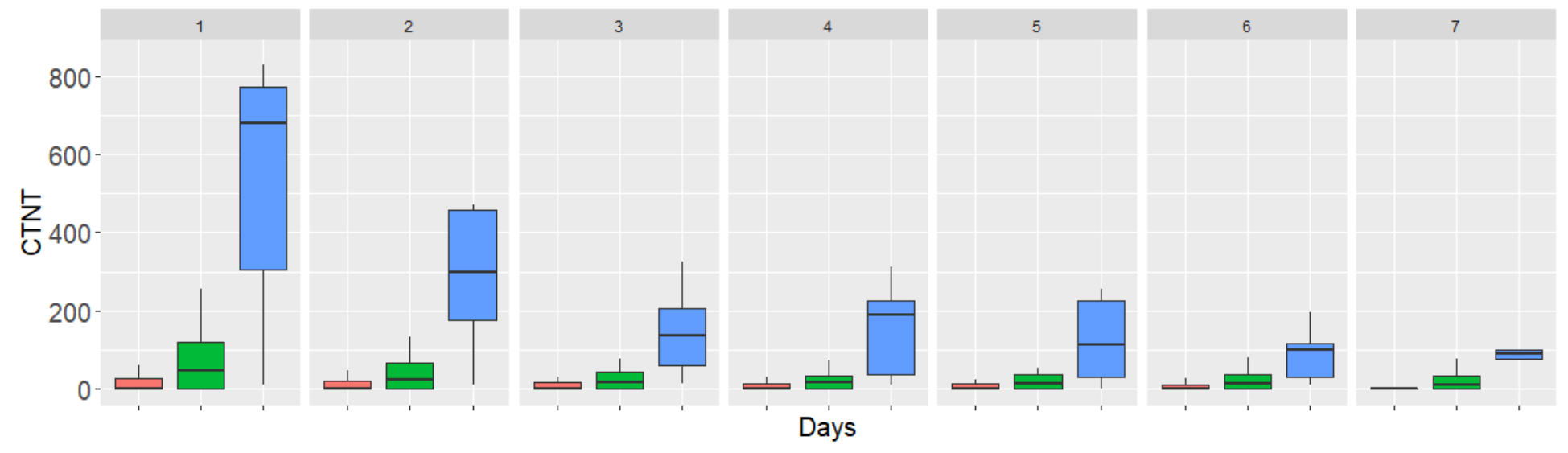

Figure 2

Differences in cardiac troponin T (CTNT) between study groups from day 1 to 7 . Boxplots show the median, upper and lower quartiles, and minimum and maximum values, with outliers omitted for clarity. Differences are highly significant each day $(p<0.001)$.

Takotsubo study group: 早 Control 追 Moderate 追 Severe

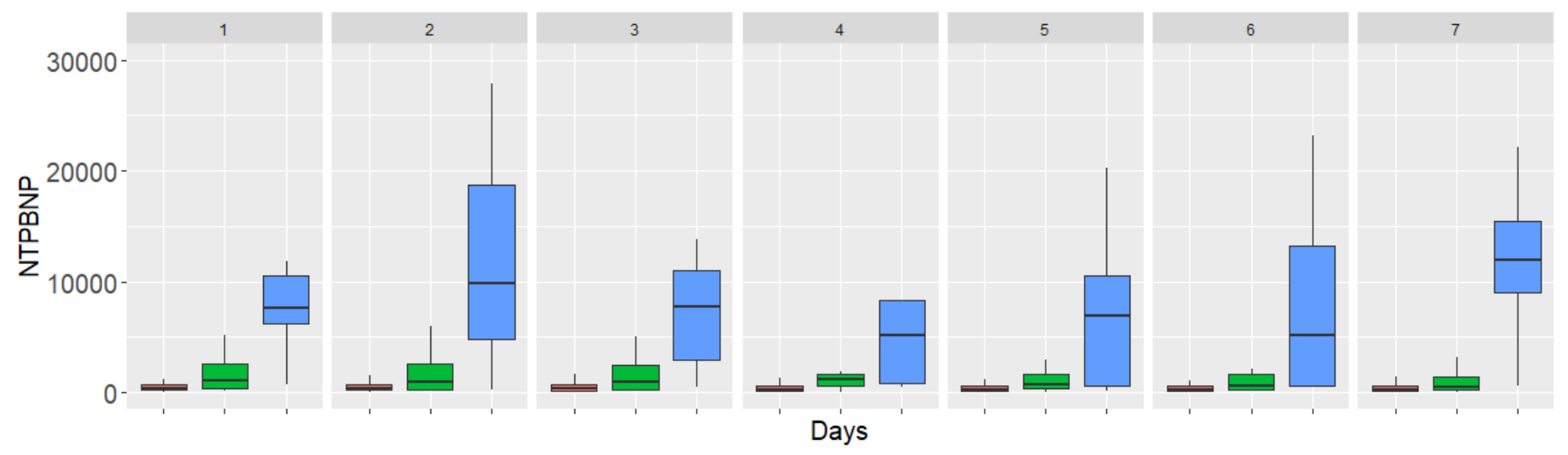

Figure 3

Differences in N-terminal pro-brain-type natriuretic peptide (NTPBNP) between study groups from day 1 to 7. Boxplots show the median, upper and lower quartiles, and minimum and maximum values, with outliers omitted for clarity. Differences are highly significant each day $(p<0.001)$. 

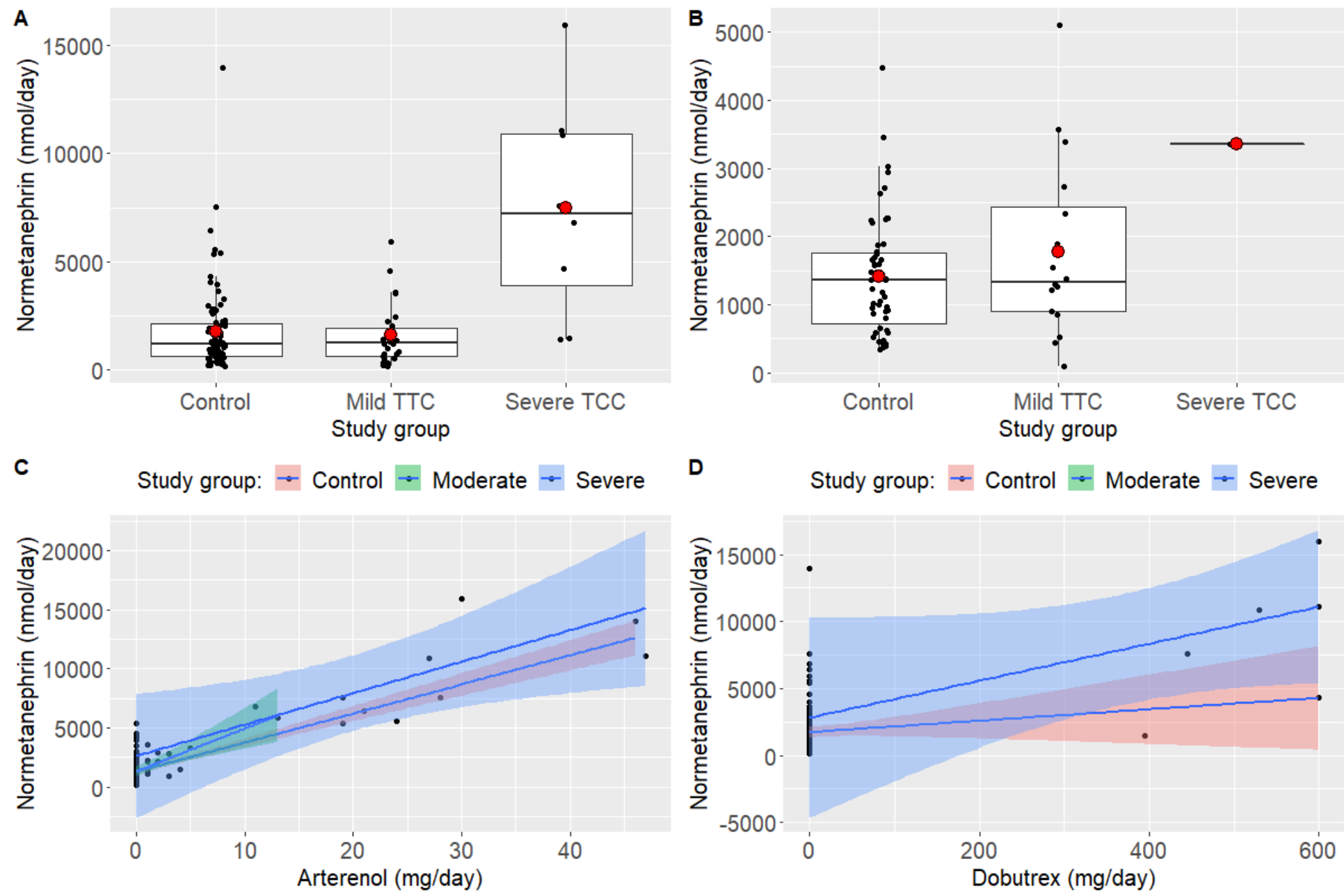

\section{Figure 4}

Urine normetanephrin levels on the day of admission (A) and 30 days later (B), and the effect of arterenol (C) and dobutamin (dobutrex) (D) on normetanephrin concentrations on the day of admission. Boxplots show datapoints (black dots jittered and outliers omitted for clarity), median, upper and lower quartiles, and minimum and maximum values; red dots indicate means. Shaded areas in C and D indicate $95 \%$ confidence intervals. Note that the scales are different on the ordinates; on the day of admission (A), median normetanephrine concentration is 7-fold higher in the severe TTC group. 

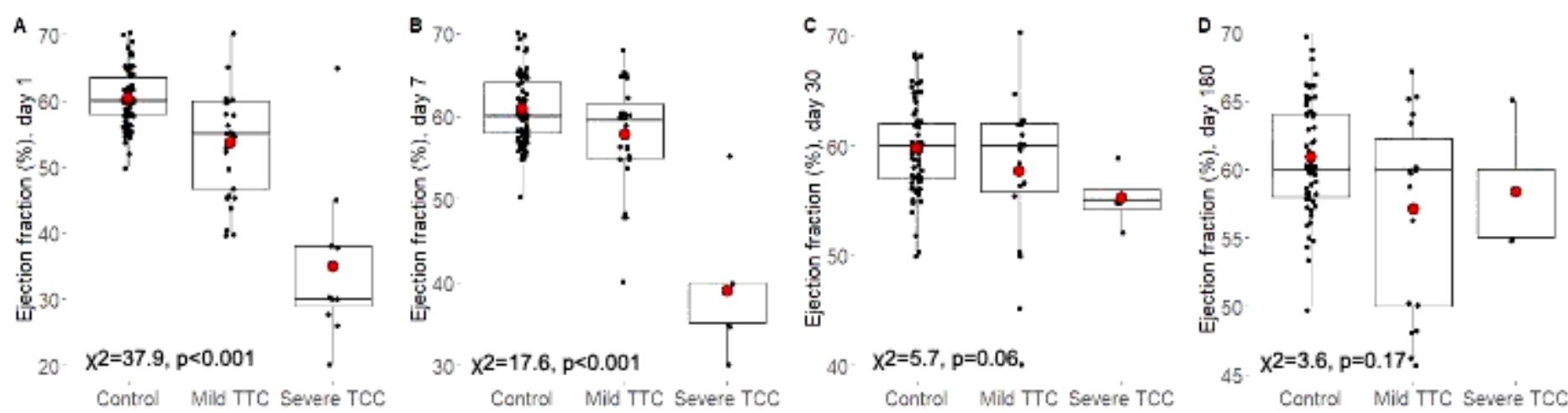

$X 2=5.7, p=0.06$
Control Mild TTC Severe TCC $45 . x^{2}=3.6, p=0.17$.

Control Mid TTC Severe TCC
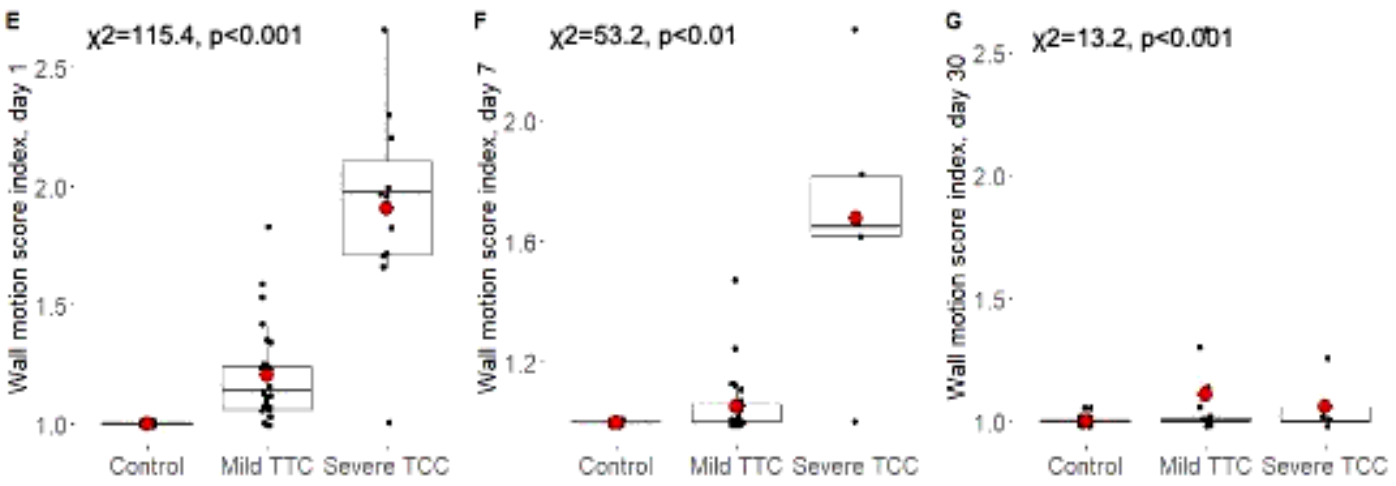

Control Mid TTC Severe TCC

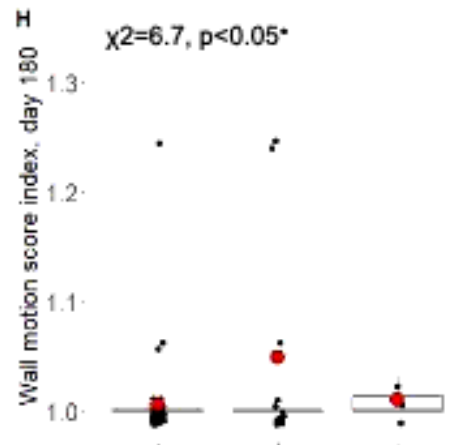

Figure 5

Differences in ejection fraction (\%) (A-D) and wall motion score index (E-H) in the study groups on day 1 , day 7, day 30 and day 180. Boxplots show datapoints (black dots; jittered and outliers omitted for clarity), median, upper and lower quartiles, and minimum and maximum values; red dots indicate means.
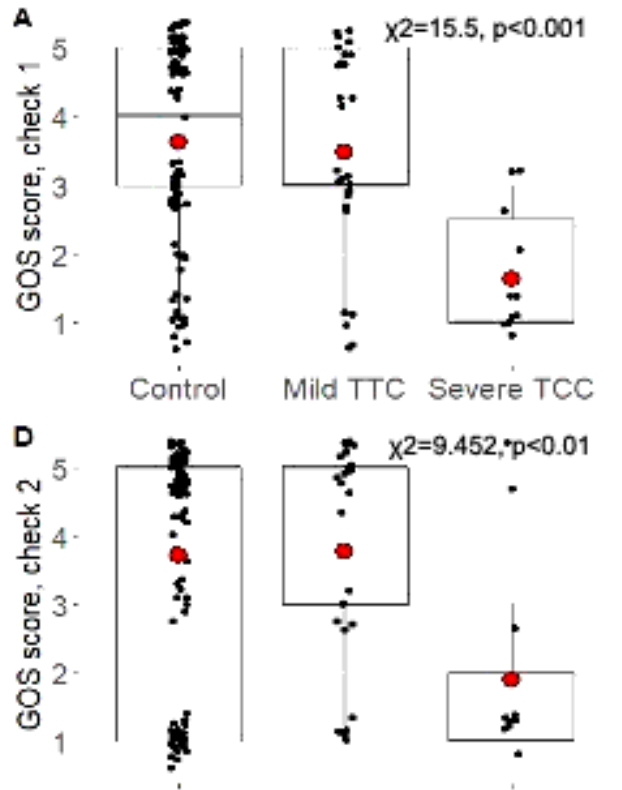

Control Mild TTC Severe TCC
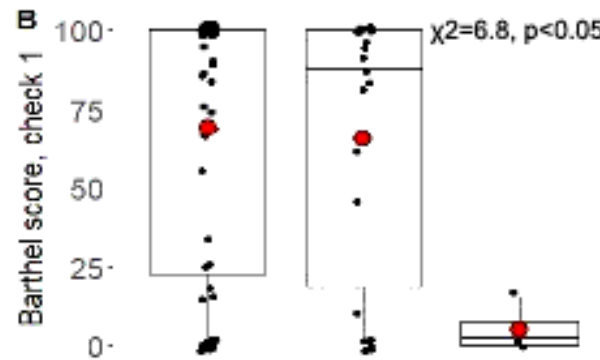

Control Mild TTC Severe TCC

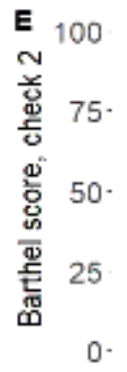

0 .

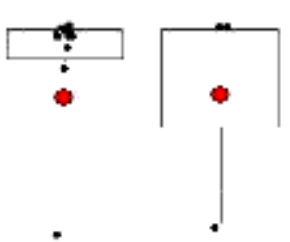

* $\quad x^{2}=1.6, p=0.44$

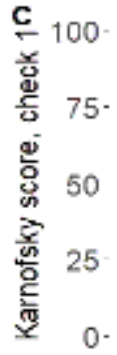

Control Mild TTC Severe TCC

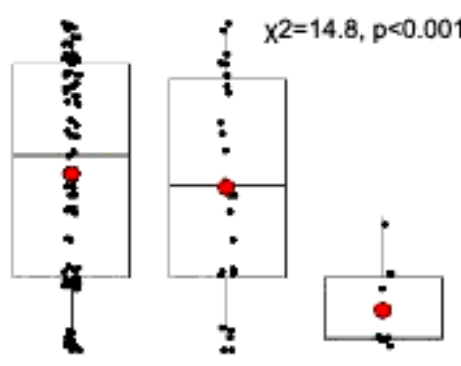

Control Mild TTC Severe TCC

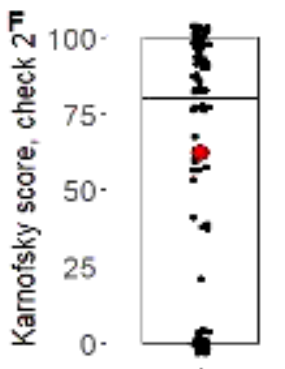

Control Mild TTC Severe TCC

Figure 6 
Differences in GOS, Barthel and Karnofsky scores between study groups at the first check-up at 30 days (A-) and the second check-up at 180 days (D-F). Boxplots show datapoints (black dots; jittered and outliers omitted for clarity), median, upper and lower quartiles, and minimum and maximum values; red dots indicate means.

\section{Supplementary Files}

This is a list of supplementary files associated with this preprint. Click to download.

- Graphicalabstract.pptx

- Supplementaltables.docx 